\title{
Heterogeneous cellular automata subway station personnel evacuation model based on cooperative behavior
}

\author{
Jie Zhuang ${ }^{1}$, Chao Wang ${ }^{1}$, a , Yongjiang $\mathrm{He}^{1}$, and Runze Song ${ }^{1}$ \\ ${ }^{1}$ College of Transportation, Shandong University of Science and Technology, Qingdao 266590, China
}

\begin{abstract}
In order to study the influence of cooperative behavior in the evacuation process of subway station personnel, and considering the heterogeneity of evacuees, the heterogeneous cellular automata method is adopted to establish a human evacuation model of subway station under cooperative behavior based on the floor field model. In the research process, the evacuated persons are divided into two types, which are seeking cooperation and accepting cooperation. Then, the effects of different cooperative behavior probability ratios of seeking cooperative personnel on evacuation efficiency, evacuation process, and evacuation bottleneck are analyzed through simulation. The result shows that cooperative behavior can effectively improve evacuation efficiency of the subway station, but it is limited by cooperative probability and the proportion of people seeking cooperation; Cooperative behavior plays a role in the whole evacuation process, which is mainly reflected in the later stage of evacuation and will promote the gathering of evacuees. The higher the probability of cooperation, the shorter the evacuation bottleneck formation time, the duration, and overall evacuation time, which will help improve the emergency safety of subway stations.
\end{abstract}

\section{Introduction}

Group evacuation is a complex and changeable process with many influencing factors with people and the environment, which may affect the evacuation process. At present, the research mainly focuses on the improvement of the pedestrian dynamic model to adapt to more complex scene simulation and extract pedestrian movement rules through experiments. They widely used simulation evacuation models like the lattice gas model, the social force model, and the cellular automata model. In 2001, Burstedde [1] first proposed the FF model, which can simulate the collective effect and self-organization phenomenon in pedestrian dynamics. Busted and Schadschneider $[2,3,4]$ improved the FF model respectively, and the improved model can effectively reproduce various typical phenomena of pedestrian flow. A. Vara [5] introduces panic parameters and random selection to simulate the process of pedestrian evacuation in a fixed obstacle room, to solve the conflict in the pedestrian location update. Zijian Fu and Xiaodong Zhou [6] established a multi-velocity field cellular automata model with less CPU time, it can simulate the crowd evacuation with dense and small speed change. Wei Guo [7] proposed an improved dynamic floor field model based on non-uniform bosons. The conversion mechanism of bosons determines the characteristics of the population system, and the improved model has more real pedestrian behavior. Gao Guoping and Guan Changsheng [8]

\footnotetext{
${ }^{\mathrm{a}}$ Corresponding author: wangchaojlu@126.com
}

proposed an evacuation cellular automata model to analyze the influence of helping behavior on evacuation efficiency and the time characteristics of the evacuation process. Chen Changkun and Tong Yunhe [9] combined the decision-making and the emotional infection theory to build the pedestrian decision-making correction model in a panic state and used the model to study the effect of panic emotion on crowd evacuation behavior. Yao Jialin and Long Shun [10] analyzed the influence of conformity behavior and environmental familiarity at different periods on the evacuation efficiency of subway personnel, to dynamically simulate the pedestrian exit selection behavior. Song Yinghua and Zhang Yu [11] proposed a cellular automata model based on avoidance behavior, which can truly simulate the effects of interaction between different groups of people.

The above studies mainly focus on the influence of panic parameters, herd behavior, help behavior, and avoidance behavior on pedestrian evacuation. Moreover, most of the existing studies are homogeneous field cellular automata models, which ignore the heterogeneity of evacuees and cannot truly simulate the movement process of crowds. From the perspective of the real scene, when an emergency at the subway station occurs, the movement behavior of the personnel will be affected by the characteristics of the event, the surrounding environment, personal psychology, physiology, and other factors, and make different evacuation behaviors. Due to the individual speed differences of humans, some people can not follow the same speed of the group and can not evacuate quickly. 
They are a kind of group in urgent need of other people's help in the evacuation process.

Considering the individual differences in the evacuation group, in this paper, we analyzed the cooperative role of different evacuees and established a heterogeneous cellular automata based personnel evacuation model, it can explore the role of cooperative behavior on the evacuation process and provide theoretical support for emergency evacuation in subway stations.

\section{Construction of evacuation model}

Evacuation is the process of continuous information interaction between a person and the outside world. The cooperative behavior between people has a certain impact on the efficiency of evacuation. In order to study the role of cooperative behavior in the evacuation, a heterogeneous cellular automata model was built to simulate the cooperative behavior in the evacuation.

To simulate pedestrian evacuation behavior more truly, the evacuation model in this paper adopts the twodimensional cellular grid. Evacuees are divided into seeking for cooperation and accepting cooperation. Moor neighborhood is adopted in cellular areas, and the probability matrix of pedestrian movement is determined accordingly, as shown in Figure 1.

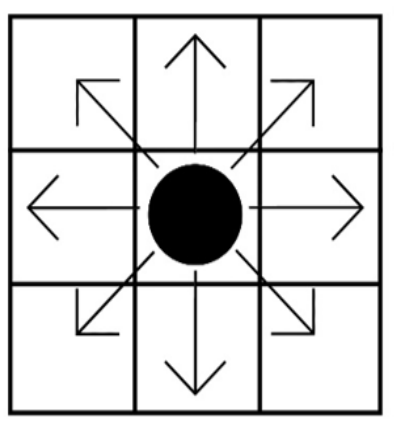

(a)

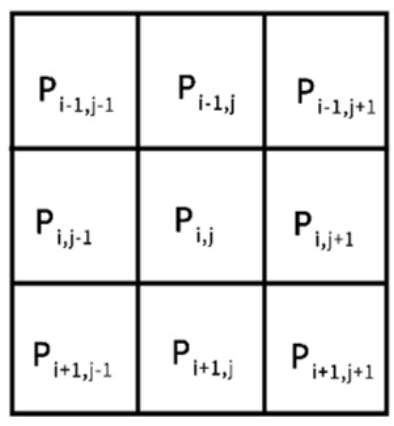

(b)
Figure 1. (a) Moore neighborhood (b) Probability matrix of evacuees movement

\subsection{Static field setting method}

The static field $S_{i, j}$ represents the distance between the evacuee and the exit in the space. The closer the distance is, the higher the value is assigned, and the higher the probability of the person's movement is. Therefore, the person will move towards the exit.

$$
S_{i, j}=r_{\max }-\sqrt{\left(x_{e}-i\right)^{2}+\left(y_{e}-j\right)^{2}}
$$

where $r_{\max }$ is the distance between the cell farthest from the exit in the evacuation space and the exit, $(i, j)$ is the coordinate value of the cell at the moment, $\left(x_{e}, y_{e}\right)$ is the coordinate value of the exit cell.

\subsection{Dynamic field setting method}

The dynamic field is the generation, diffusion, and influence of evacuation information among evacuees.

$$
D_{i, j}^{n}(t+1)=f\left(D_{i, j}^{n}(t), \alpha, \beta\right)+\mu \Delta D
$$

The diffusion and attenuation of the dynamic field $f\left(D_{i, j}^{n}(t), \alpha, \beta\right)$ are discretized respectively 12 to obtain the updating rules of the dynamic field:

$$
\begin{array}{r}
D_{i, j}^{n}(t+1)=(1-\alpha)(1-\beta) D_{i, j}^{n}(t)+ \\
\frac{\alpha(1-\beta)}{8}\left[D_{i-1, j-1}^{n}(t)+D_{i-1, j}^{n}(t)+D_{i-1, j+1}^{n}(t)+\right. \\
D_{i, j-1}^{n}(t)+D_{i, j+1}^{n}(t)+D_{i+1, j-1}^{n}(t)+ \\
\left.D_{i+1, j}^{n}(t) D_{i+1, j+1}^{n}(t)\right]+\mu \Delta D
\end{array}
$$

where $\alpha$ is the diffusion coefficient and $\beta$ is the attenuation coefficient. When a pedestrian leaves the cell $(i, j)$ at the last moment, $\mu=1$, and the dynamic field value of the cell increases $\Delta D$; otherwise, $\mu=0$.

\subsection{Cooperative field setting method}

Considering the role of cooperation between people, this paper introduces a cooperation field similar to the static field in the heterogeneous FF model [12], as shown in Figure 2. Attach a value to each yellow square, the red square is the person seeking cooperation, and then the value of each square is the reciprocal of the distance to the red square. The closer the distance, the greater the value. This value is proportional to the probability, so the higher the value, the greater the probability of moving to the red square.

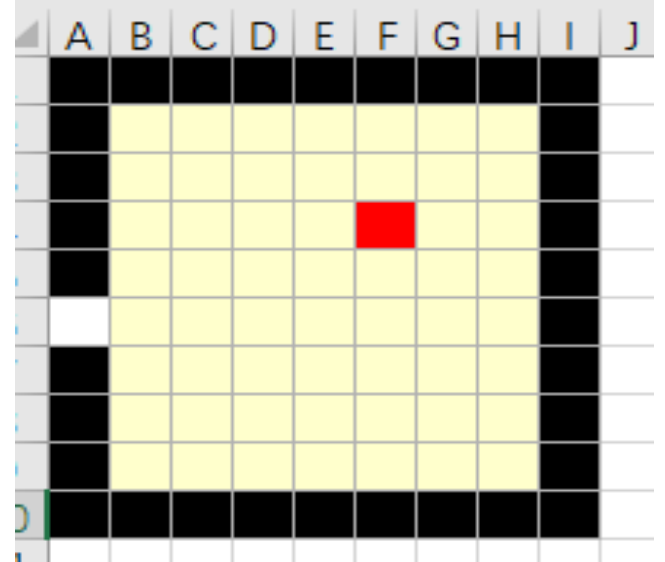

Figure 2. Cooperative field setting

$$
\mathrm{A}_{i, j}=\left\{\begin{aligned}
1, & h_{i, j}=1 \\
\sum_{h_{i, j}=1} \frac{1}{\sqrt{(x-i)^{2}+(y-\mathrm{j})^{2}}}, & h_{i, j}=0
\end{aligned}\right.
$$

where $h_{i, j}$ is the ability of personnel to seek cooperation, If cooperation is required, $h_{i, j}=1$; otherwise, $h_{i, j}=0$; $g_{i, j}$ is the ability of people to accept cooperation, if cooperate, $g_{i, j}=1$, otherwise, $g_{i, j}=0$; According to the probability of cooperation $y$ set whether to accept the cooperation; $\mathrm{r}$ is the maximum distance of cooperation, $(i, j)$ is the coordinate value of the cell at the moment, and $(x, y)$ is the coordinate value of the cell that needs cooperation.

\subsection{Simulation update rule}

The mobile probability of the cell is: 


$$
\begin{gathered}
P_{\left(i_{0}, j_{0}\right) \rightarrow(i, j)}(t)=N^{-1} \times\left[\operatorname { e x p } \left[-K_{s}\left(S_{i, j}-S_{i_{0}, j_{0}}\right)+\right.\right. \\
\left.K_{D}\left(D(t)_{i, j}-D(t)_{i_{0}, j_{0}}\right)+K_{A} A_{i, j}\right] \times\left(1-\eta_{i, j}\right) \times \\
\left.\xi_{i, j} \times\left(1-g_{i, j}\right)+\exp \left[K_{A}\left(A_{i, j}-A_{i_{0}, j_{0}}\right)\right] \times g_{i, j}\right](5)
\end{gathered}
$$

where $N$ is to ensure $\sum_{(i, j)} P_{i j}$ normalized factor, $K_{S}, K_{D}, K_{A}$ are respectively static field parameters, dynamic field parameters, and cooperative field parameters; $S_{i, j}$ is the static field value; $D_{i, j}^{n}(t)$ is the dynamic field value, and $\eta_{i j}$ is the state recognition factor of the target cell on the cell $(i, j)$. If the target cell is occupied by pedestrians at the current moment, then $\eta_{i j}=1$, otherwise, $\eta_{i j}=0 . \xi_{i j}$ is the type identification factor of the cell $(i, j)$. If the cell $(\mathrm{I}, \mathrm{j})$ is a building, then $\xi_{i j}=0$, otherwise, $\xi_{i j}=1$.

$\mathrm{N}$ is expressed as:

$$
\begin{array}{r}
N=\sum_{i, j} \exp \left[-K_{S}\left(S_{i, j}-S_{i_{0}, j_{0}}\right)+K_{D}\left(D(t)_{i, j}-\right.\right. \\
\left.\left.D(t)_{i_{0}, j_{0}}\right)+K_{A} A_{i, j}\right] \times\left(1-\eta_{i, j}\right) \times \xi_{i, j} \times\left(1-g_{i, j}\right)+ \\
\exp \left[K_{A}\left(A_{i, j}-A_{i_{0}, j_{0}}\right)\right] \times g_{i, j}
\end{array}
$$

The status update of evacuees adopts parallel update rules. Simulation steps and update rules are as follows:

(1) The location of evacuees (random distribution in cellular space) is initialized and the static field parameter $K_{S}$ is calculated, the dynamic field parameter $K_{D}$ and cooperative field parameter $K_{A}$ are set, and the evacuation time is 0 .

(2) According to formula (5), the transfer probability of each evacuee is calculated, and the target cell is selected according to $P_{(i, j)}$.

(3) When multi-person movement conflicts, the dynamic field rules, and cooperative field rules are updated according to $E q$. (3) (4).

(4) Clear the evacuees in the exit area and make $t=$ $t+\Delta t$ at the same time.

(5) When all the evacuees in the cellular space evacuate, the simulation ends, otherwise go back to step (2) to recalculate.

\section{Simulation and result analysis}

\subsection{Simulation model parameter setting}

Using MATLAB to simulate the model, set the cell size as $0.4 \times 0.4 \mathrm{~m}^{2}$, The system update time step is $\Delta t=0.3 \mathrm{~s}$, pedestrians can only move one cell at a time. The maximum moving speed of pedestrians during the simulation is about $1.33 \mathrm{~m} / \mathrm{s}$, the speed of pedestrians seeking cooperation is $0.333 \mathrm{~m} / \mathrm{s}$, the common speed after cooperation is $0.667 \mathrm{~m} / \mathrm{s}$. The evacuation simulation model is established in a $100 \times 80$ cell space with an exit width of $0.4 \mathrm{~m}$, as shown in Figure 3. Set $K_{s}=2, K_{D}=2$, $\Delta D=0.2, \alpha=0.4, \beta=0.7, \mathrm{r}=2.5 \mathrm{~m}$, and the proportion of people seeking cooperation is a.

Since the initial positions of pedestrians in each experiment are randomly distributed, to eliminate the influence of random distribution, the experiments under different cooperation probability parameters are repeated 30 times, and the evacuation time and number are recorded, and the average value is analyzed.

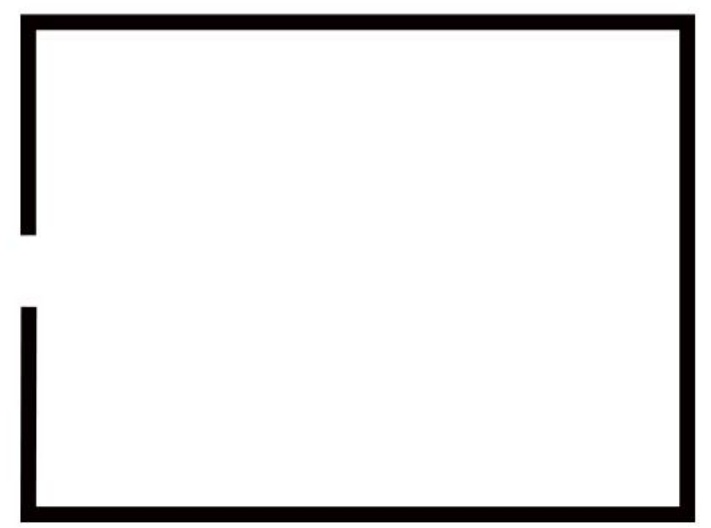

Figure 3. Cellular space

\subsection{The influence of cooperative behavior on evacuation efficiency}

The evacuation efficiency is analyzed under different proportions of people seeking cooperation and the different probability of cooperation, as shown in Figure 4.

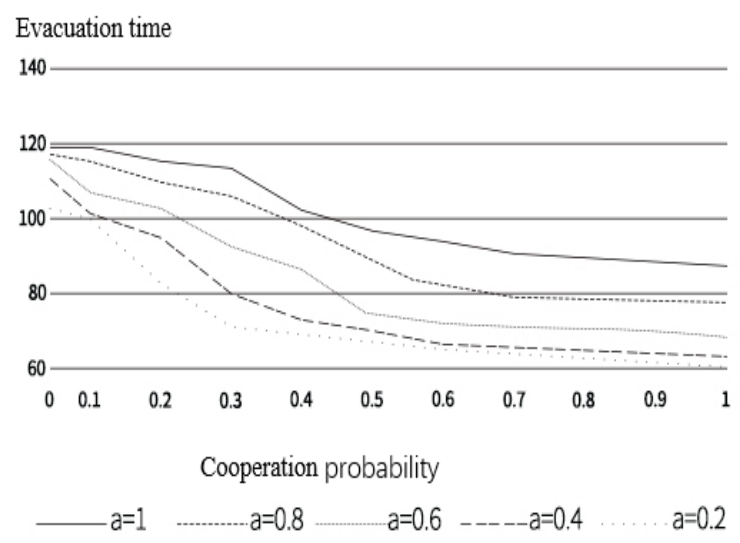

Figure 4. Evacuation time under different $y$

As the cooperation probability $y$ increases, the overall curve shows a downward trend. The higher the proportion of people seeking cooperation, the longer the evacuation time. When $\mathrm{a}=0.4, y>0.3$ and $\mathrm{a}=0.6, y>0.5$, the evacuation effect is more significant. When $y$ getting great, the curve tends to be flat, and the increase in evacuation efficiency is not obvious. The reason is that cooperative behavior gradually reaches saturation. The increase of $y$ can effectively improve the evacuation efficiency but is limited by $a$. This result is consistent with the actual situation. When the number of people seeking cooperation reaches a certain proportion, the supply exceeds the demand, the overall movement speed is reduced, and the evacuation time is extended.

\subsection{The influence of cooperative behavior on the evacuation process}


It can be seen from Figure 5 that cooperative behavior has an impact on the whole evacuation process. From Figure 5 $a, b, c$, it can be seen that at the beginning of the evacuation, and the slope of $y$ is greater when it is lower, which shows that the evacuation efficiency is higher at that time. With the evacuation process going on, when the time is greater than $t_{1}$, the slope of low $y$ is less than that of high $y$, and the value of $t_{1}$ is gradually increasing with the proportion of people seeking cooperation. In the later stage of evacuation, the increase of $y$ promotes the cooperative behavior and improves the overall evacuation speed. Therefore, the slope and the evacuation efficiency in the Figure 5 is higher.

\subsection{The influence of cooperative behavior on evacuation bottleneck}

The evacuation bottleneck will be formed in the exit area during the evacuation process. The curve of evacuation personnel density changing with time in the exit area is simulated to explore the influence of different cooperation probability on the evacuation bottleneck. The simulation results are shown in Figure 6 . With the increase of evacuation time, limited to the exit width, the evacuation personnel gathers in the exit area and the density is gradually increased. Under the same cooperation probability, the peak density of the export area is basically the same, about 4 people per square meter. With the greater of the cooperation probability, the evacuation time to reach the peak density and the duration will shorter, and the overall evacuation time will less. The personnel movement scenario in Figure 7 can more intuitively show the impact of cooperation probability on the evacuation bottleneck. In scenes with high cooperation probability, evacuees move to the exit faster and the evacuation bottleneck forms earlier. Due to the limitation of the exit width, the evacuation efficiency is more significant in the exit area with a fan distribution.



Number of people

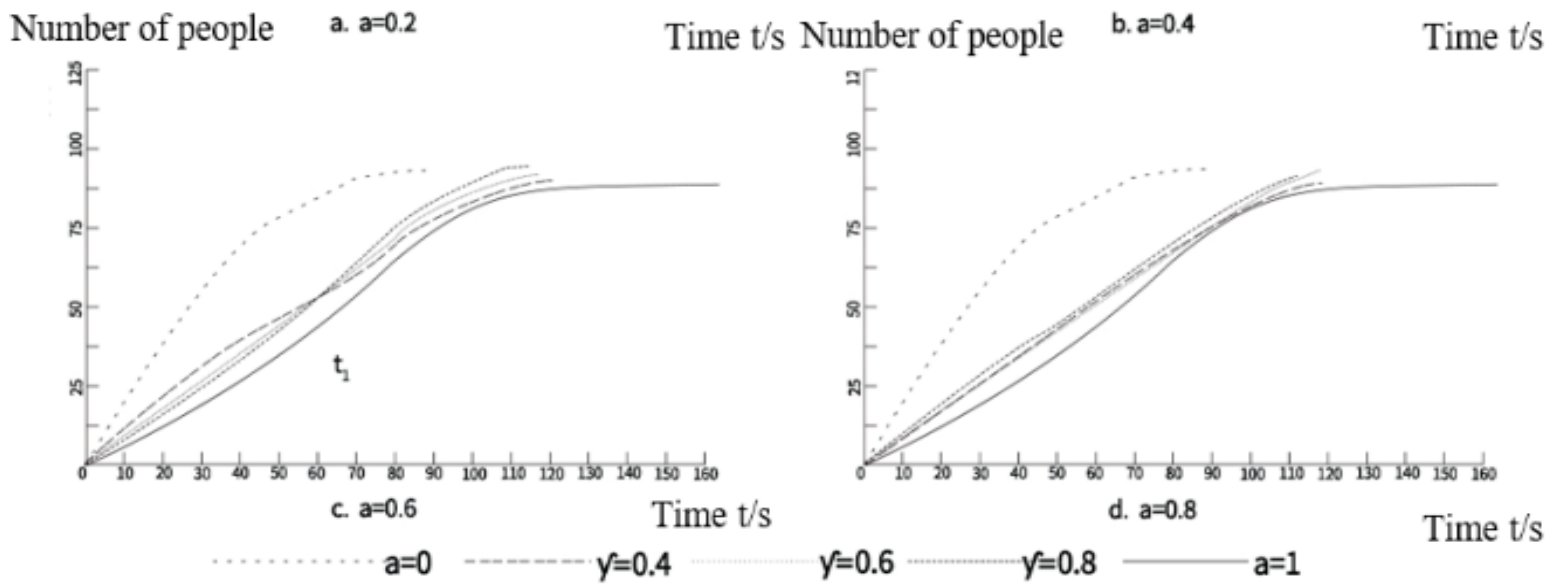

Figure 5. Relationship between evacuation and time

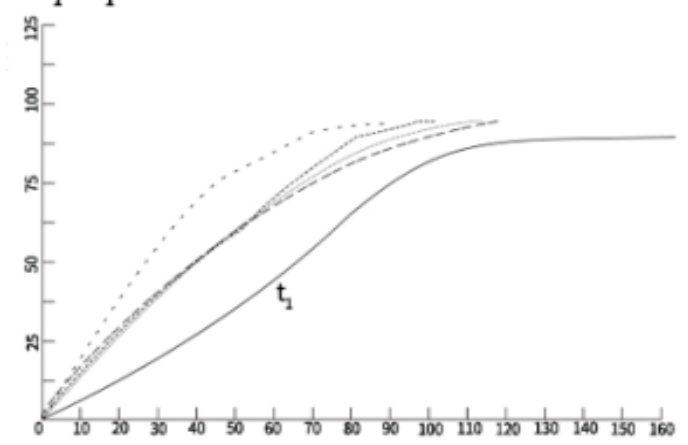




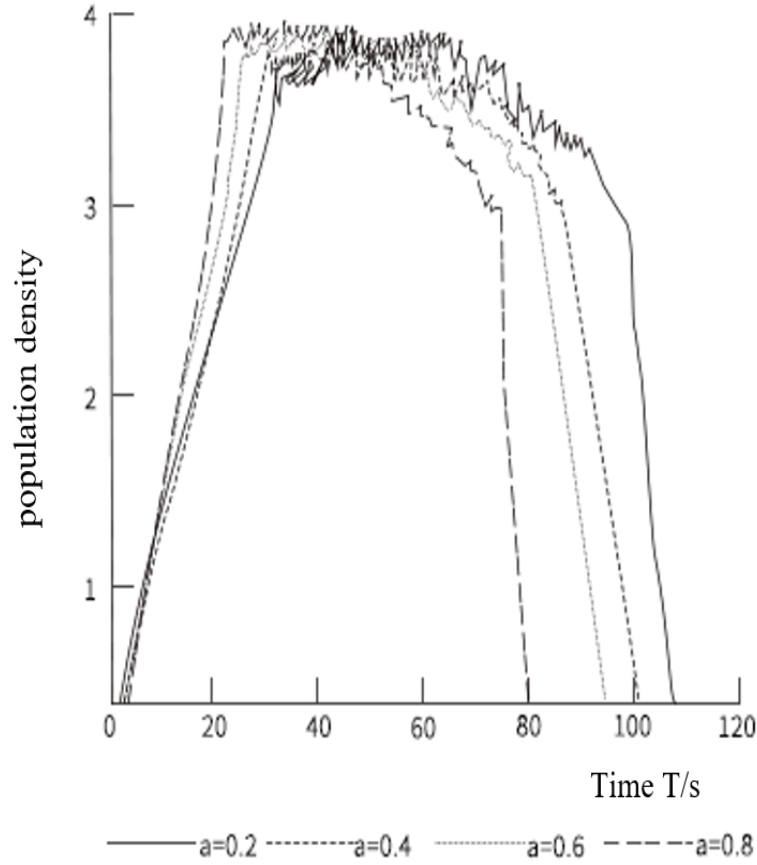

Figure 6. Export area density
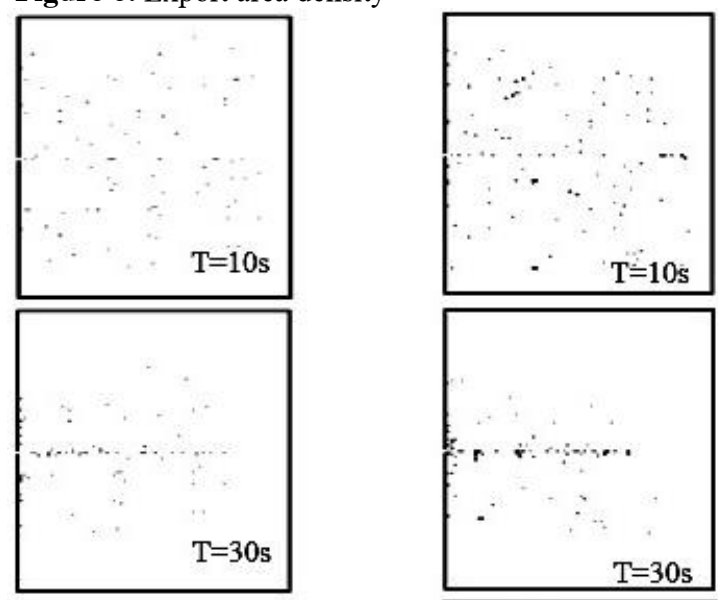



$y=0.4$

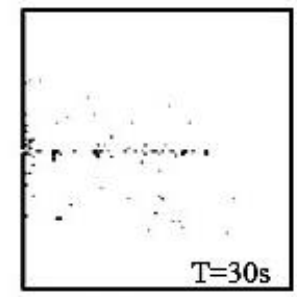

$y=0.6$

Figure 7. Personnel movement scenario

\section{Conclusion}

1) Cooperative behavior can effectively improve the evacuation efficiency of subway stations, but affected by the probability of cooperation and the people seeking cooperation, in a certain range, the higher the proportion of people seeking cooperation, the more significant the role of cooperative behavior in improving evacuation efficiency, the better the emergency security.

2) Cooperative behavior also plays a role in the whole evacuation process. When the proportion of people seeking the cooperation of the station is a certain low degree, the influence of cooperative behavior is mainly reflected in the later stage of the emergency evacuation.

3 ) The exit area of the subway is the bottleneck area in the whole evacuation process. Cooperative behaviors can accelerate the gathering of evacuees towards the exit direction of the subway station and significantly shorten the duration of the bottleneck and improve the efficiency of emergency evacuation.

\section{References}

1. Burstedde C, Klauck K, Schadschneider A, et al. Simulation of pedestrian dynamics using a twodimensional cellular automaton. Physica A: Statistical Mechanics and its Applications, 295(3): 507-525, (2001).

2. Burstedde C, Kirchner A, Klauck K, et al. Cellular automaton approach to pedestrian dynamics-applications. arXiv preprint condmat/0112119, (2001).

3. Schadschneider A. Cellular automaton approach to pedestrian dynamics-theory. arxiv preprint cond-mat/0112117, (2001).

4. Schadschneider A, Klingsch W, Klüpfel H, et al. Evacuation dynamics: Empirical results, modeling and application//Extreme Environmental Events. Springer New York, $517-$ 550, (2001).

5. A. Varas, M.D. Cornejo, D. Mainemer, et al. Cellular automaton model for evacuation process with obstacles. Physica A 382: 631-642,(2007).

6. Zh.J. Fu, X.D. Zhou, K.J. Zhu, et al. A floor field cellular automaton for crowd evacuation considering different walking abilities. Physica A 420: 294-303, (2015).

7. W. Guo, X.L. Wang, M.T. Liu, et al. Modification of the dynamic floor field model by the heterogeneous bosons. Physica A 417: 358-366, (2015).

8. G.P. Gao, C.S. Guan. A helping behaviorconsidering cellular automaton model for evacuation from a building. China Safety Science Journal, 28(1): 56-61, (2018).

9. C.H. Chen, Y.H. Tong. Study on crowd evacuation model under panic state based on cellular automata. Journal of Safety Science and Technology, 15(6): 12-17, (2019).

10. J.L. Yao, S. Long. Pedestrian evacuation simulation in metro station based on cellular automata. Journal of Railway Science and Engineering,16(11):2897-2902, (2019).

11. Y.H. Song, Y. Zhang, F.Z. Zhou, et al. Study on Evacuation Cellular Automaton Model Considering Avoidance Behavio. Journal of System Simulation,32(06):975-981, (2020).

12. Chao Wang, Jian Wang. A Modified Floor Field Model Combined with Risk Field for Pedestrian Simulation. Mathematical Problems in Engineering, DOI: 10.1155/2016/9653860, (2016). 